\title{
THE RIEMANN PROBLEM FOR AN ELASTIC STRING WITH A LINEAR HOOKE'S LAW
}

\author{
BY \\ HARALD HANCHE-OLSEN (Department of Mathematical Sciences, The Norwegian University of \\ Science and Technology, N-7491 Trondheim, Norway), \\ HELGE HOLDEN (Department of Mathematical Sciences, The Norwegian University of Science \\ and Technology, N-7491 Trondheim, Norway),
}

AND

\begin{abstract}
NILS HENRIK RISEBRO (Department of Mathematics, University of Oslo, P.O. Box 1053, Blindern, N-0316 Oslo, Norway)
\end{abstract}

\begin{abstract}
We solve the Riemann problem for vibrations of an infinite, planar, perfectly elastic string with a linear relation between the string tension and the local stretching, i.e., with a linear Hooke's law. The motion is governed by a $4 \times 4$ system of conservation laws that is linearly degenerate in all wave families. Conservation of energy leads to $L^{2}$ estimates that are used in the analysis.
\end{abstract}

1. Introduction. The mathematics of vibrations of elastic strings is a truly classical problem with the first step being taken by the Pythagoreans, relating the harmonious vibrations of elastic strings with their relative lengths. However, the first description of vibrations of elastic strings in terms of differential equations was given by Taylor in 1713 . He derived the fundamental frequency of the stretched vibrating string. Euler derived in 1744 the correct equation for large vibrations of a planar string. The solution in 1746 by d'Alembert of the problem of small transversal vibrations of an elastic string, which reduces to the familiar one-dimensional (linear) wave equation, initiated a fundamental discussion of the foundation of mathematical analysis, in particular the concept of a function, and was instrumental in the development of analysis. Over the years, various aspects - analytical, numerical, and applications - of the wave equation have been studied, and it has remained a cornerstone in partial differential equations. We will make no

Received March 21, 2000.

2000 Mathematics Subject Classification. Primary 35L65.

Key words and phrases. Elastic string, Riemann problem, Hooke's law.

Research supported in part by The Norwegian Research Council.

E-mail address: hanche@math.ntnu.no

WWW address: http://www .math.ntnu.no/ hanche/

E-mail address: holden@math.ntnu.no

WWW address: http://www.math.ntnu.no/ holden/

E-mail address: nilshr@math.uio.no 
attempt to discuss this extensive development further, but rather concentrate on results relevant for our approach.

In this paper we will study the Riemann problem for large vibration of a planar, elastic string with a linear stress-strain relationship (Hooke's law). Shearer solved the Riemann problem for fairly general nonlinear stress-strain relationships in [11] and [12]. While his assumptions exclude the linear case treated here, the framework applies equally well in the linear and nonlinear cases. See also [10], [3], and [15]. Li, Serre, and Zhang [9] studied the "generalized Riemann problem" in the linear case that we analyze here, and obtained a local solution in time except in certain critical cases. However, we obtain a very complete and global solution of the Riemann problem, covering all cases.

Our purpose is to give a self-contained exposition of these results for the case of a string satisfying Hooke's law. This special case derives some interest from the fact that the resulting system, though linearly degenerate in all wave families, exhibits nonlinear phenomena. We conclude that the solution is unique by using conservation of energy.

Let us, before we describe our results and how they relate to other results, introduce our notation. We consider planar motion of an infinite, elastic string, and let $x \in \mathbb{R}$ denote the arc coordinate of a material point on the string in a reference position at rest. At any time $t$ we let the position of the point $x$ on the string be denoted by $(X(x, t), Y(x, t)) \in \mathbb{R}^{2}$. The velocity is given by

$$
u(x, t)=X_{t}(x, t), \quad v(x, t)=Y_{t}(x, t)
$$

(We use subscripts to indicate partial derivatives: $X_{t}=\partial X / \partial t$, etc.) Assume that we have a tension $T(x, t)$ at the point $x$ and time $t$ acting tangentially along the string, and the absence of any body forces on the string. Newton's law on differential form can be written as

$$
\left(\frac{T X_{x}}{\sqrt{\left(X_{x}\right)^{2}+\left(Y_{x}\right)^{2}}}\right)_{x}=X_{t t}, \quad\left(\frac{T Y_{x}}{\sqrt{\left(X_{x}\right)^{2}+\left(Y_{x}\right)^{2}}}\right)_{x}=Y_{t t}
$$

where we have assumed a homogeneous string, and so disposed of the constant density of the unstrained string (which should have appeared as a factor on the right-hand side) by subsuming it into $T$. As we will see later, these equations have to be interpreted in the weak sense. The local strain is $r-1$, where

$$
r=\sqrt{\xi^{2}+\eta^{2}}
$$

and

$$
\xi(x, t)=X_{x}(x, t), \quad \eta(x, t)=Y_{x}(x, t) .
$$


Writing the system of two second order equations above as a first order system of four conservation laws, we find

$$
\begin{array}{r}
u_{t}-\left(\frac{T \xi}{r}\right)_{x}=0 \\
v_{t}-\left(\frac{T \eta}{r}\right)_{x}=0 \\
\xi_{t}-u_{x}=0 \\
\eta_{t}-v_{x}=0
\end{array}
$$

in which the first two equations express the conservation of momentum, and the latter two the conservation of mass. For the Riemann problem we are given initial data

$$
(u(x, 0), v(x, 0), \xi(x, 0), \eta(x, 0))= \begin{cases}\left(u_{L}, v_{L}, \xi_{L}, \eta_{L}\right), & x<0 \\ \left(u_{R}, v_{R}, \xi_{R}, \eta_{R}\right), & x>0\end{cases}
$$

A very careful and lucid discussion of the derivation of the equations for large vibrations of strings, as well as some historical background, is given by Antman [1], [2]. See also Weinberger [14].

The assumption that the string is perfectly elastic is contained in the relation that the tension depends only on the local strain, i.e.,

$$
T=T(r) \text {. }
$$

Furthermore, we shall assume

$$
T(1)=0, \quad T^{\prime}(r)>0, \quad T^{\prime}(1)=1,
$$

i.e., there is no tension in the unstrained string, and tension increases with strain; the final equation can be ensured by suitable rescaling.

The linear wave equation as a model for small transversal vibrations of an elastic string is obtained from the assumption that $\xi>1$ is nearly constant, and $\eta$ is small. In this paper we will solve the Riemann problem (2), (3), for a string with a linear stress-strain relationship $T(r)=r-1$ and a suitable class of initial data. For brevity we write

$$
U_{t}+F(U)_{x}=0, \text { with } U=\left(\begin{array}{c}
u \\
v \\
\xi \\
\eta
\end{array}\right) \text { and } F(U)=-\left(\begin{array}{c}
T \xi / r \\
T \eta / r \\
u \\
v
\end{array}\right)
$$

A simple calculation (see next section) reveals that the matrix $F^{\prime}(U)$ has four real, distinct eigenvalues; thus the system of conservation laws is strictly hyperbolic. Two of the eigenvalues correspond to transversal waves (with characteristic speeds $\pm \sqrt{1-r^{-1}}$ ) and two correspond to longitudinal waves (with characteristic speeds \pm 1 ). The characteristic speeds were computed by [6], [7, pp. 206ff]. The eigenvalues $\lambda_{i}$ and corresponding eigenvectors $e_{i}$ of the Jacobian $F^{\prime}(U)$ satisfy

$$
\nabla \lambda_{i} \cdot e_{i}=0, \quad i=1, \ldots, 4
$$

and thus the system is linearly degenerate in all families. 
Keyfitz and Kranzer [8] considered the string with a nonlinear stress-strain relationship in which $T^{\prime \prime}(r)>0$. In that setting, the transversal waves made up a linearly degenerate family, while the longitudinal waves formed a non-degenerate family. As we have seen, both of these families become linearly degenerate for a linear law, and so both families support contact discontinuities.

In addition to the contact discontinuities, we find certain anomalous shocks corresponding to a $180^{\circ}$ bend in the string, but these shocks either create or destroy energy. For this reason, and because the Riemann problem has a unique solution without any anomalous shocks, we dismiss the anomalous shocks as unphysical.

2. Elementary waves. In this section we investigate the elementary waves for the system of equations (2) under the assumptions (1), (1). We first wish to calculate the rarefaction curves of $(2)$; therefore we consider smooth solutions, for which (2) can be written in quasilinear form as

$$
\frac{\partial}{\partial t}\left(\begin{array}{c}
u \\
v \\
\xi \\
\eta
\end{array}\right)-\left(\begin{array}{cccc}
0 & 0 & \frac{\xi^{2}}{r^{2}} T^{\prime}+\frac{\eta^{2}}{r^{3}} T & \frac{\xi \eta}{r^{2}} T^{\prime}-\frac{\xi \eta}{r^{3}} T \\
0 & 0 & \frac{\xi \eta}{r^{2}} T^{\prime}-\frac{\xi \eta}{r^{3}} T & \frac{\eta^{2}}{r^{2}} T^{\prime}+\frac{\xi^{2}}{r^{3}} T \\
1 & 0 & 0 & 0 \\
0 & 1 & 0 & 0
\end{array}\right) \frac{\partial}{\partial x}\left(\begin{array}{c}
u \\
v \\
\xi \\
\eta
\end{array}\right)=0
$$

in which the $4 \times 4$ matrix (the Jacobian $\left.F^{\prime}(U)\right)$ is of the special form $-\left(\begin{array}{ll}0 & A \\ I & 0\end{array}\right)$ with

$$
A=T^{\prime} R R^{\mathrm{T}}+\frac{T}{r} \Theta \Theta^{\mathrm{T}}, \quad R=\left(\frac{\xi}{r}, \frac{\eta}{r}\right)^{\mathrm{T}}, \quad \Theta=\left(-\frac{\eta}{r}, \frac{\xi}{r}\right)^{\mathrm{T}} .
$$

Clearly, the matrix $A$ has eigenvalues $T^{\prime}$, with eigenvector $R$, and $T / r$, with eigenvector $\Theta$. Whenever $\lambda^{2}$ is an eigenvalue of $A$ with eigenvector $a,\left(\begin{array}{ll}0 & A \\ I & 0\end{array}\right)$ has the eigenvalue $\lambda$ with eigenvector $\left(\begin{array}{c}\lambda a \\ a\end{array}\right)$. Thus we obtain the following eigenvalues and eigenvectors of the Jacobian

$$
\lambda= \pm \sqrt{T^{\prime}}: \quad\left(\begin{array}{c}
\mp \xi \\
\mp \eta \\
\xi \\
\eta
\end{array}\right), \quad \lambda= \pm \sqrt{T / r}: \quad\left(\begin{array}{c} 
\pm \sqrt{T / r} \eta \\
\mp \sqrt{T / r} \xi \\
-\eta \\
\xi
\end{array}\right)
$$

We may note that, for the most realistic stress-strain relationships, $0<T / r<T^{\prime}$ for all $r>1$, so the system is strictly hyperbolic in this region. When $r=1$, however, the two middle eigenvalues $\pm \sqrt{T / r}$ coincide - and when $r<1$, they become imaginary, rendering the system unstable.

Computing the rarefaction curves, i.e., the integral curves of the eigenvector fields, is a trivial exercise. For the middle eigenvalues $\pm \sqrt{T / r}$ we see that the $(\xi, \eta)$ projections of these curves are circles centered at the origin. Hence $r$, and hence the eigenvalues themselves, are constant along these curves, and we conclude that the middle families are linearly degenerate. In the case of the outer eigenvalues $\pm \sqrt{T / r}$, however, the $(\xi, \eta)$ projections of the rarefaction curves are straight line segments through the origin - and so the outer families are linearly degenerate if, and only if, the stress-strain relationship is linear - i.e., $T^{\prime}$ is constant, or Hooke's law holds. 
In the remainder of this paper we will concentrate on the string with a linear stressstrain relationship; see [5], [4], [6], [7, pp. 206ff]. Thus we assume

$$
T(r)=r-1,
$$

the normalization following from (1), and let

$$
\sigma=\sqrt{T / r}=\sqrt{1-\frac{1}{r}} .
$$

The eigenvalues and their corresponding eigenvectors can be rewritten as

$$
\lambda= \pm 1: \quad\left(\begin{array}{c}
\mp \xi \\
\mp \eta \\
\xi \\
\eta
\end{array}\right), \quad \lambda= \pm \sigma: \quad\left(\begin{array}{c} 
\pm \sigma \eta \\
\mp \sigma \xi \\
-\eta \\
\xi
\end{array}\right)
$$

We also see that for $r \geq 1, \sigma$ takes values in the interval $[0,1\rangle$, so that the system (2) is hyperbolic in $r \geq 1$ and strictly hyperbolic in $r>1$. Fig. 1 shows the eigenvectors with the corresponding eigenvalues in the $(\xi, \eta)$ plane and the $(u, v)$ plane.
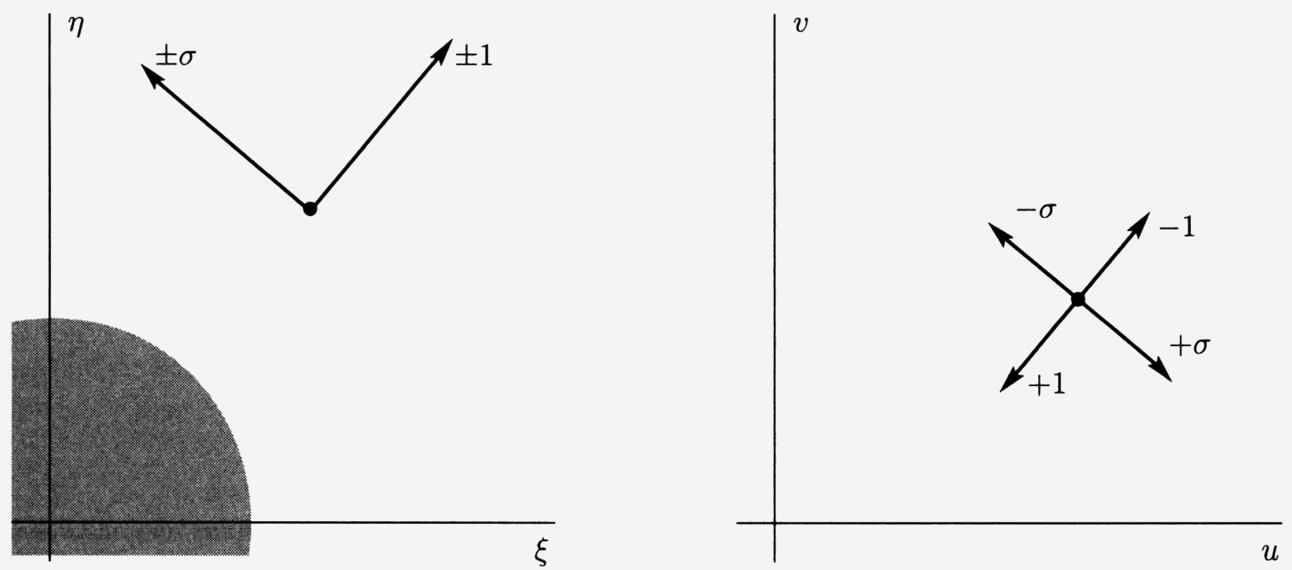

FIG. 1. The $\xi \eta$ and $u v$ projections of the eigenvectors

The rarefaction curves are the integral curves of the eigenvector fields. For the $\pm \sigma$ eigenvalues, the corresponding differential equations are

$$
u^{\prime}= \pm \sigma \eta, \quad v^{\prime}=\mp \sigma \xi, \quad \xi^{\prime}=-\eta, \quad \eta^{\prime}=\xi
$$

with the solution $\left(u_{R}(\theta), v_{R}(\theta), \xi_{R}(\theta), \eta_{R}(\theta)\right)$ given by

$$
\begin{array}{ll}
\xi_{R}(\theta)=r \cos \left(\theta_{L}+\theta\right), & \eta_{R}(\theta)=r \sin \left(\theta_{L}+\theta\right), \\
u_{R}(\theta)=u_{L} \mp \sigma r\left(\cos \left(\theta_{L}+\theta\right)-\cos \theta_{L}\right), & v_{R}(\theta)=v_{L} \mp \sigma r\left(\sin \left(\theta_{L}+\theta\right)-\sin \theta_{L}\right),
\end{array}
$$

where $\left(\xi_{L}, \eta_{L}\right)=\left(r \cos \theta_{L}, r \sin \theta_{L}\right)$. The resulting curves are indicated in Fig. 2. Note that the two wave curves have the same projection in the $\xi \eta$-plane, while they have different projections in the $u v$-plane. When the $\xi \eta$-projection travels counterclockwise 

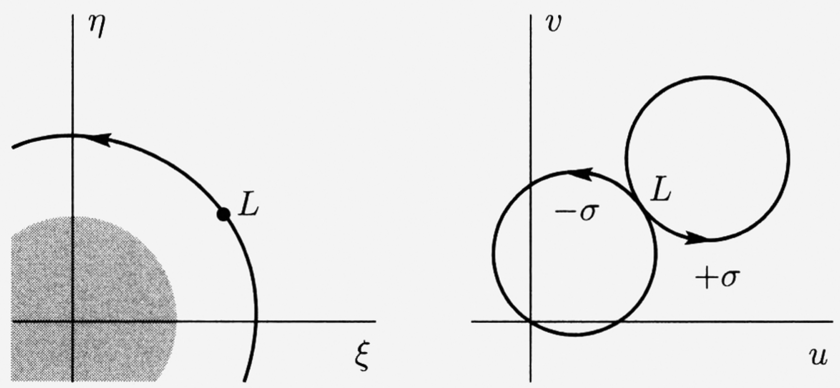

Fig. 2. Wave curves for $s= \pm \sigma$.

around the circle, the $u v$-projection travels counterclockwise around the corresponding circle as well.

The differential equations for the \pm 1 eigenvalues are

$$
u^{\prime}=\mp \xi, \quad v^{\prime}=\mp \eta, \quad \xi^{\prime}=\xi, \quad \eta^{\prime}=\eta,
$$

with the solution $\left(u_{R}(\zeta), v_{R}(\zeta), \xi_{R}(\zeta), \eta_{R}(\zeta)\right)$ given by

$$
\xi_{R}(\zeta)=e^{\zeta} \xi_{L}, \quad \eta_{R}(\zeta)=e^{\zeta} \eta_{L}, \quad u_{R}(\zeta)=u_{L} \mp\left(e^{\zeta}-1\right) \xi_{L}, \quad v_{R}(\zeta)=v_{L} \mp\left(e^{\zeta}-1\right) \eta_{L},
$$

valid for $r_{L} e^{\zeta} \geq 1$, where $r_{L}=\sqrt{\xi_{L}^{2}+\eta_{L}^{2}}$. This parametrizes a line whose $\xi \eta$-projection is the part of the line through the origin and $\left(\xi_{L}, \eta_{L}\right)$ extending from the circle $r=1$ via $\left(\xi_{L}, \eta_{L}\right)$ to infinity; and whose $u v$-projection is parallel to the $\xi \eta$-projection and passes through $\left(u_{L}, v_{L}\right)$. Furthermore, as $\left(\xi_{R}, \eta_{R}\right)$ travels along its line, $\left(u_{R}, v_{R}\right)$ travels equally far in the opposite direction (for the +1 eigenvalue) or the same direction (for the -1 eigenvalue) - see Fig. 3.
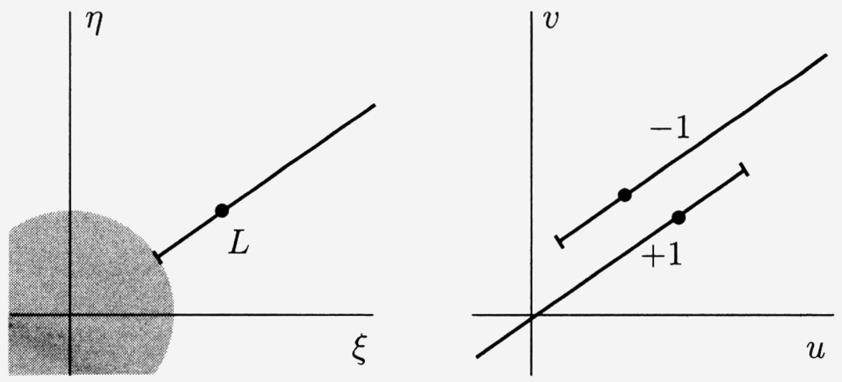

FIG. 3. Wave curves for $s= \pm 1$. For clarity's sake, we show wave curves through different states with the same $(\xi, \eta)$ components but slightly different $(u, v)$ components.

Next we calculate the Hugoniot loci of (2). Since all wave families are linearly degenerate, we already know [13] that the rarefaction curves through a given state are part of the Hugoniot locus of that state, with the corresponding discontinuities being contact discontinuities.

However, we shall compute the Hugoniot locus explicitly anyway, to make sure we do not miss any part of it. Let subscripts $L$ and $R$ denote the state to the left and 
right of a shock respectively, and let [·] denote the jump across a discontinuity, i.e., $[\phi(U)]=\phi\left(U_{R}\right)-\phi\left(U_{L}\right)$ for any function $\phi=\phi(U)$. If $s$ denotes the speed of the shock, then the Hugoniot condition reads

$$
\begin{aligned}
s[u]+\left[\sigma^{2} \xi\right] & =0, & s[v]+\left[\sigma^{2} \eta\right] & =0, \\
s[\xi]+[u] & =0, & s[\eta]+[v] & =0,
\end{aligned}
$$

which we write more conveniently in the equivalent form

$$
\begin{aligned}
s^{2}[\xi] & =\left[\sigma^{2} \xi\right], & s^{2}[\eta] & =\left[\sigma^{2} \eta\right], \\
{[u] } & =-s[\xi], & {[v] } & =-s[\eta],
\end{aligned}
$$

reducing the task ahead to solving the two-dimensional problem (4) and substituting the answer in (5).

When $r_{R}=r_{L}$, then also $\sigma_{R}=\sigma_{L}$, so (4) reduces to $s^{2}=\sigma^{2}$, and we recover the contact discontinuities with $U_{R}$ along the $\pm \sigma$-rarefaction waves through $U_{L}$.

If, on the other hand, $r_{R} \neq r_{L}$, we use the relation $\left[\sigma^{2} \xi\right]=\sigma_{R}^{2}[\xi]+\left[\sigma^{2}\right] \xi_{L}$ and obtain from (4)

$$
\left(s^{2}-\sigma_{R}^{2}\right)\left(\begin{array}{l}
{[\xi]} \\
{[\eta]}
\end{array}\right)=\left[\sigma^{2}\right]\left(\begin{array}{c}
\xi_{L} \\
\eta_{L}
\end{array}\right)
$$

which, since $\left[\sigma^{2}\right] \neq 0$, implies that the point $\left(\xi_{R}, \eta_{R}\right)$ lies on the straight line through $(0,0)$ and $\left(\xi_{L}, \eta_{L}\right)$. Using $\sigma^{2}=1-1 / r$ we can rewrite $(4)$ as

$$
\left(s^{2}-1\right)[\xi]=\left[\frac{\xi}{r}\right], \quad\left(s^{2}-1\right)[\eta]=\left[\frac{\eta}{r}\right] .
$$

If $\left(\xi_{R}, \eta_{R}\right)$ lies on the ray from the origin through $\left(\xi_{L}, \eta_{L}\right)$ then the right-hand sides of the above equations vanish, and so (6) reduces to $s= \pm 1$. Thus we recover the contact discontinuities with $U_{R}$ along the \pm 1 -rarefaction waves through $U_{L}$.

If, on the other hand, $\left(\xi_{R}, \eta_{R}\right)$ lies on the opposite ray, then $[\xi / r]=2 \xi_{L} / r_{L}$ and $[\eta / r]=2 \eta_{L} / r_{L}$, and substituting this into (6) and rearranging we get

$$
\xi_{R}=\left(1+\frac{2}{r_{L}\left(s^{2}-1\right)}\right) \xi_{L}, \quad \eta_{R}=\left(1+\frac{2}{r_{L}\left(s^{2}-1\right)}\right) \eta_{L},
$$

in which the parentheses must be negative for consistency's sake. Thus

$$
r_{R}=-\left(1+\frac{2}{r_{L}\left(s^{2}-1\right)}\right) r_{L}
$$

which we can solve for $s$ to get

$$
s^{2}=1-\frac{2}{r_{L}+r_{R}} .
$$

We shall see later that the Riemann problem can always be solved without using these kinds of shock waves, and also that energy is not conserved across such shocks. We shall therefore refer to these shocks as anomalous shocks. However, note that the anomalous shock curve intersects the $\pm \sigma$ wave curve at the point where $r_{R}=r_{L}-$ i.e., at $\left(\xi_{R}, \eta_{R}\right)=\left(-\xi_{L},-\eta_{L}\right)$. Thus, this particular discontinuity is not counted among the anomalous shocks. In other words, an anomalous shock is associated with the string bending back on itself (a $180^{\circ} \mathrm{kink}$ ) with a jump in tension across the discontinuity - 
a sort of combination of a transversal wave and a longitudinal one. While one may feel somewhat uncomfortable with such waves, the energy argument at the end of the next section provides a better justification for rejecting them.

3. The Riemann problem. Having calculated the Hugoniot loci, we now show that the Riemann problem has a unique solution, barring anomalous shocks, consisting of at most five constant states separated by discontinuities. Given two states $U_{L}$ and $U_{R}$ such that $r_{L} \geq 1$ and $r_{R} \geq 1$, we consider the initial value problem

$$
\begin{gathered}
U_{t}+F(U)_{x}=0, \\
U(x, 0)= \begin{cases}U_{L} & \text { for } x \leq 0, \\
U_{R} & \text { for } x>0 .\end{cases}
\end{gathered}
$$

We wish to find states $U_{1}, U_{2}$, and $U_{3}$ such that

$$
U_{L} \stackrel{C_{-1}}{\longrightarrow} U_{1} \stackrel{C_{-\sigma}}{\longrightarrow} U_{2} \stackrel{C_{\sigma}}{\longrightarrow} U_{3} \stackrel{C_{1}}{\longrightarrow} U_{R}
$$

where the mapping $C_{s}$ denotes a contact discontinuity with speed $s$, as found in the previous section.

Since the angle in the $(\xi, \eta)$ plane remains constant under the mappings $C_{ \pm 1}$, and the radius in the $(\xi, \eta)$ plane remains constant under the mappings $C_{ \pm \sigma}$, and remembering that $[u]=-s[\xi]$ and $[v]=-s[\eta]$, we really have only two unknowns in the Riemann problem: the $\xi$ and $\eta$ components of the middle state $U_{2}$; see Fig. 4. Clearly, any choice of $\left(\xi_{2}, \eta_{2}\right)$ would be admissible, except that the $(u, v)$ components would not match. This yields two equations to determine the two unknowns.
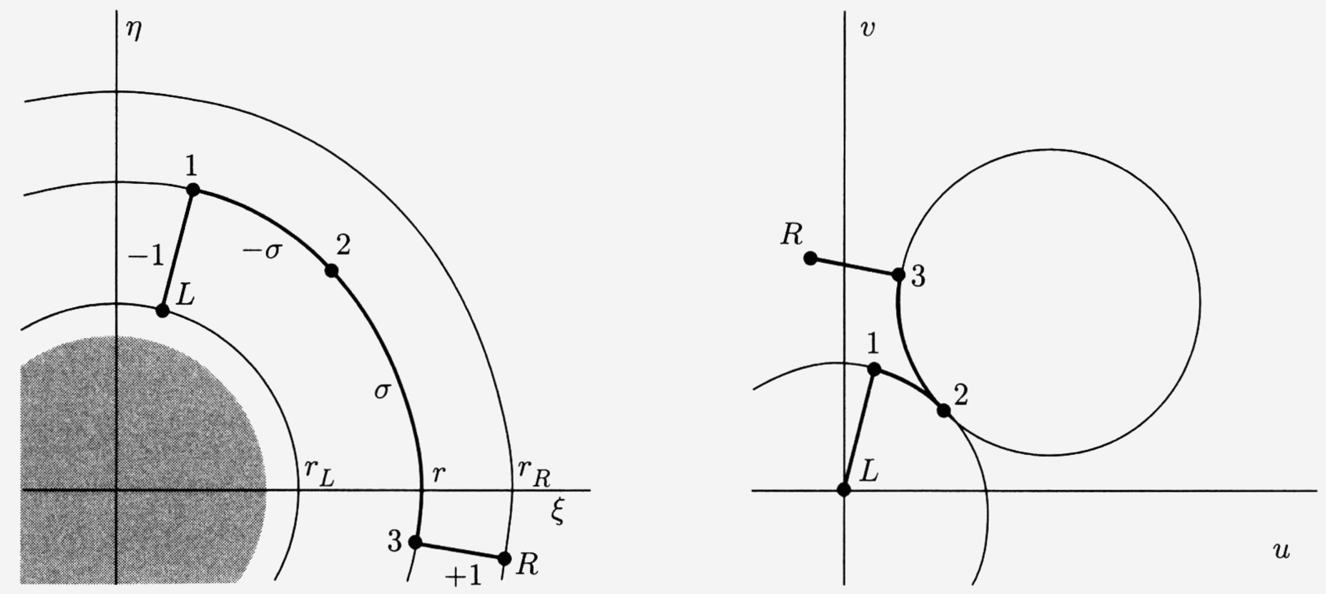

FIG. 4. Solution path in the $\xi \eta$-plane and in the $u v$-plane. Note that the solution only involves the five points indexed; $L, 1,2,3$, and $R$; the curves shown are included for orientation only. 
In the following we let $(r, \theta)$ denote polar coordinates in the $(\xi, \eta)$ plane. In order to find the middle state we calculate $u_{R}$ by using (5):

$$
\begin{aligned}
u_{R} & =u_{L}+\left(u_{1}-u_{L}\right)+\left(u_{2}-u_{1}\right)+\left(u_{3}-u_{2}\right)+\left(u_{R}-u_{3}\right) \\
& =u_{L}+\left(\xi_{1}-\xi_{L}\right)+\sigma\left(\xi_{2}-\xi_{1}\right)-\sigma\left(\xi_{3}-\xi_{2}\right)-\left(\xi_{R}-\xi_{3}\right) \\
& =u_{L}-\xi_{R}-\xi_{L}+(1-\sigma)\left(\xi_{1}+\xi_{3}\right)+2 \sigma \xi_{2},
\end{aligned}
$$

which we prefer to write as

$$
\underbrace{u_{R}-u_{L}+\xi_{R}+\xi_{L}}_{2 \alpha}=(1-\sigma) r_{2}(\underbrace{\cos \theta_{L}+\cos \theta_{R}}_{2 \gamma})+2 \sigma \xi_{2} .
$$

We have the similar equation for the $v$ component:

$$
\underbrace{v_{R}-v_{L}+\eta_{R}+\eta_{L}}_{2 \beta}=(1-\sigma) r_{2}(\underbrace{\sin \theta_{L}+\sin \theta_{R}}_{2 \delta})+2 \sigma \eta_{2} .
$$

Dropping the subscript 2 and introducing $\alpha-\delta$ as indicated above, we therefore have two equations for the unknowns $\xi$ and $\eta$, with $r=\sqrt{\xi^{2}+\eta^{2}}$ and $\sigma=\sqrt{1-1 / r}$ :

$$
\begin{aligned}
& \alpha=\gamma r(1-\sigma)+\sigma \xi \\
& \beta=\delta r(1-\sigma)+\sigma \eta .
\end{aligned}
$$

We shall show that these equations have a unique solution $(\xi, \eta)$. To this end, consider (8) as defining a map $(\xi, \eta) \mapsto(\alpha, \beta)$. This function maps a circle of radius $r$ and centered at the origin in the $\xi \eta$-plane to a circle $\Gamma(r)$ of radius $R(r)=r \sigma$ and centered at $P(r)=r(1-\sigma)(\gamma, \delta)$ in the $\alpha \beta$-plane (but note that $\Gamma(1)$ degenerates to the point $(\gamma, \delta))$. Noting that $R^{\prime}>1$ for $r>1, R(1)=0$, and $R(r) \rightarrow \infty$ as $r \rightarrow \infty$, while $|P(r)|$ is bounded, we conclude that the union of the circles $\{\Gamma(r): r \geq 1\}$ covers the entire $\alpha \beta$-plane. Furthermore, since $\sigma_{r}>0$ we find $(r \sigma)_{r}>1$, and since $\gamma^{2}+\delta^{2} \leq 1$ we get $\left|P^{\prime}\right| \leq(r \sigma)_{r}-1$ while $R^{\prime}=(r \sigma)_{r}>\left|P^{\prime}\right|$, from which it follows that all the circles $\{\Gamma(r): r \geq 1\}$ are disjoint. Hence the map $(\xi, \eta) \mapsto(\alpha, \beta)$ maps the region $r>1$ one-to-one onto $\mathbb{R}^{2} \backslash\{(\gamma, \delta)\}$, and (8) always has a solution $(\xi, \eta)$ which is unique unless $(\alpha, \beta)=(\gamma, \delta)$, in which case all $(\xi, \eta)$ on the circle $r=1$ are solutions. This ambiguity has no consequences for the solution of the Riemann problem, however. For when $r=1$ then $\sigma=0$, so the two waves of speed $\pm \sigma$ coincide, and the value of the middle state $U_{2}$ is of no consequence.

We define the energy as

$$
e=\frac{1}{2}\left(u^{2}+v^{2}+(r-1)^{2}\right) .
$$

This can be viewed as kinetic energy plus "potential energy" stored in the stretching of the string. For smooth solutions, we compute

$$
\begin{aligned}
e_{t} & =u u_{t}+v v_{t}+(r-1) r_{t} \\
& =u\left(\frac{(r-1) \xi}{r}\right)_{x}+v\left(\frac{(r-1) \eta}{r}\right)_{x}+(r-1) \frac{\xi u_{x}+\eta v_{x}}{r} \\
& =\left(\frac{r-1}{r}(u \xi+v \eta)\right)_{x}
\end{aligned}
$$


so that energy is conserved with a flux given by

$$
\Phi=-\sigma^{2}(u \xi+v \eta)
$$

We now show that the energy is also conserved over the contact discontinuities $C_{s}$, but not over the anomalous discontinuities. Let $P$ be defined as $P=s[e]-[\Phi]$, where $s$ is the speed of the discontinuity. Let first $s= \pm 1$. Thanks to the rotation invariance of the equations, we may assume without loss of generality that $\left(\xi_{L}, \eta_{L}\right)$ and $\left(\xi_{R}, \eta_{R}\right)$ are located on the positive $\xi$-axis, so that in particular $v_{L}=v_{R}$ and $r=\xi$.

$$
\begin{aligned}
P & =s[e]-[\Phi]=s\left[\frac{1}{2}(\xi-1)^{2}+\frac{1}{2} u^{2}+\frac{1}{2} v^{2}\right]-\left[\left(\frac{1}{\xi}-1\right) \xi u\right] \\
& =s((\langle\xi\rangle-1)[\xi]+\langle u\rangle[u])-[u]+\langle\xi\rangle[u]+\langle u\rangle[\xi]=0,
\end{aligned}
$$

where we used (5) at the end. Here, $\langle w\rangle$ denotes the average of the left and right states, i.e., $\frac{1}{2}\left(w_{L}+w_{R}\right)$. Similarly, for $s= \pm \sigma$, we have $[r]=0$, and

$$
\begin{aligned}
P & =s\left[\frac{1}{2} u^{2}+\frac{1}{2} v^{2}\right]+\sigma^{2}[\xi u+\eta v] \\
& =s(\langle u\rangle[u]+\langle v\rangle[v])+s^{2}(\langle\xi\rangle[u]+\langle u\rangle[\xi]+\langle\eta\rangle[v]+\langle v\rangle[\eta]) \\
& =-s^{3}(\langle\xi\rangle[\xi]+\langle\eta\rangle[\eta])=-\frac{s^{3}}{2}\left[\xi^{2}+\eta^{2}\right]=0 .
\end{aligned}
$$

Similar computations show that energy is not conserved across anomalous shocks. Again, we may assume without loss of generality that $\xi_{R}>0, \xi_{L}<0, \eta_{R}=\eta_{L}=0$. In particular $[r]=2\langle\xi\rangle$ and $[\xi]=2\langle r\rangle$. The relevant calculation (somewhat abbreviated) is now (using (7) in the last step)

$$
\begin{aligned}
P & =s\left[\frac{1}{2} u^{2}+\frac{1}{2}(r-1)^{2}\right]+\left[\left(1-\frac{1}{r}\right) u \xi\right] \\
& =s(\langle u\rangle[u]+\langle r-1\rangle[r])+\langle u\rangle[\xi]+\langle\xi\rangle[u]-2\langle u\rangle \\
& =-s^{2}\langle u\rangle[\xi]+s(\langle r\rangle-1)[r]+\langle u\rangle[\xi]-s\langle\xi\rangle[\xi]-2\langle u\rangle \\
& =2\left(\left(1-s^{2}\right)\langle r\rangle-1\right)\langle u\rangle+s(\langle r\rangle-1)[r]-s\langle r\rangle[r] \\
& =-s[r]
\end{aligned}
$$

which is nonzero for anomalous shocks. We have now proved the theorem:

Theorem 1. The Riemann problem for the system of equations (2) has a unique scale invariant solution in which the energy given by (9) is conserved, provided the initial states $U_{L}$ and $U_{R}$ are outside the cylinder $Z$ given by $\xi^{2}+\eta^{2}<1$. This solution consists of at most five constant states, separated at most by the contact discontinuities $C_{-1}$, $C_{-\sigma}, C_{\sigma}$, and $C_{1}$, and all intermediate states are outside $Z$.

\section{REFERENCES}

[1] S. S. Antman. Equations for large vibrations of strings. Amer. Math. Monthly, 87:359-370, 1980.

[2] S. S. Antman. Nonlinear Problems in Elasticity. Springer-Verlag, New York, 1995.

[3] C. Carasso, M. Rascle, and D. Serre. Étude d'un modéle hyperbolique en dynamique des câbles. M2AN Math. Model. Numer. Anal., 19:573-599, 1985.

[4] G. F. Carrier. On the non-linear vibration problem of the elastic string. Quart. J. Appl. Math., 3:157-165, 1945. 
[5] J. W. Craggs. Wave motion in plastic-elastic strings. J. Mech. Phys. Solids, 2:286-295, 1954.

[6] N. Cristescu. Spatial motion of elastic-plastic strings. J. Mech. Phys. Solids, 9:165-178, 1961.

[7] N. Cristescu. Dynamic Plasticity. North-Holland, Amsterdam, 1967.

[8] B. L. Keyfitz and H. C. Kranzer. A system of non-strictly hyperbolic conservation laws arising in elasticity theory. Arch. Rat. Mech. Anal., 72:219-241, 1980.

[9] Ta-Tsien Li, D. Serre, and Hao Zhang. The generalized Riemann problem for the motion of elastic strings. SIAM J. Math. Anal., 23:1189-1203, 1992.

[10] D. Serre. Un modèle relaxé pour les câbles inextensibles. M2AN Math. Model. Numer. Anal., 25:465$481,1991$.

[11] M. Shearer. Elementary wave solutions of the equations describing the motion of an elastic string. SIAM J. Math. Anal., 16:447-459, 1985.

[12] M. Shearer. The Riemann problem for the planar motion of an elastic string. J. Diff. Eq., 61:149$163,1986$.

[13] B. Temple. Systems of conservation laws with invariant submanifolds. Trans. Amer. Math. Soc., 280:781-795, 1983.

[14] H. F. Weinberger. A First Course in Partial Differential Equations. Xerox, Lexington, 1965.

[15] R. Young. Wave interactions in nonlinear elastic strings. University of Massachusetts, 2000. 